\title{
Tecnura
}

\section{Homeostasis de la industria de manufactura en Jalisco, México: el kaizen como negentropía en la logística de embarques}

\author{
Homeostasis of the manufacturing industry in Jalisco, México: \\ kaizen as a negentropy in shipping logistics
}

\author{
Irma Aracely Toscano Rentería ${ }^{1 \mathbb{D}}$, Esmeralda Brito Cervantes ${ }^{2}$, Santos Magaña Moya ${ }^{3}$, \\ Mario Guadalupe Gonzaléz Pérez ${ }^{4}$
}

Fecha de recepción: 9 de mayo de 2019

Fecha de aceptación: 23 de agosto de 2019

Como citar: Toscano I., A., Brito E., Magaña S. y González, M., G.y (2019). Homeostasis de la industria electrónica en Jalisco: el kaizen en la logística de embarques. Tecnura, 23(62), 21-33. https://doi.org/10.14483/22487638.15453

\section{Resumen:}

Contexto: En México, el comercio internacional experimenta un aumento al que se suman empresas internacionales del área de manufactura en el estado de Jalisco. La investigación revisa la homeostasis de las empresas de ensamble y exportación, con la idea de mejorar la logística de embarques para automatizar e incrementar la productividad, a través del desarrollo y aplicación de la herramienta de mejora continua denominada kaizen.

Método: Esta investigación parte de un enfoque sistémico. En este sentido, para identificar la homeostasis de la empresa se obtuvieron datos e información de las actividades que desarrolla el área logística, así como políticas y estrategias. Posteriormente, mediante el diagrama de Ishikawa se analizaron la relación causa-efecto y a través de un mapeo de procesos se determinó la problemática de las áreas de compra, producción y almacén. Finalmente, se plantearon varias alternativas de solución para el mejoramiento de la gestión. Asimismo, para el desarrollo de las soluciones, se confrontaron los problemas y soluciones relacionándolos con el impacto en el nivel de servicio, y se hizo un comparativo entre el antes y después de implementar el kaizen.

Resultados: El estudio se centra en el funcionamiento de la logística de embarques para automatizar el proceso y evitar los cuellos de botella. De esta manera, las ordenes que no se embarcan a tiempo se convierten en código A (órdenes urgentes) y provocan que el cliente califique un $60 \%$ la productividad. Por ello, al implementar el kaizen se logra subir considerablemente hasta en un $35 \%$ por año, con muestras de satisfacción y calificación del $95 \%$. Cabe mencionar que este kaizen se llevó a cabo en el proyecto de Fru's \& Opt.

Conclusiones: La homeostasis actual de las herramientas mencionadas en el estudio son básicas y necesarias. Igualmente, se siguen actualizando y adaptando herramientas de mejora continua

1 Licenciada en Informática Administrativa, maestra en Comercio Internacional. Universidad de Especialidades. Guadalajara, México. Contacto: irma_toscano@my.uvm.edu.mx

2 Licenciada en Mercadotecnia Internacional, maestra en Negocios y Estudios Económicos, doctora en Negocios y Estudios Económicos. Universidad de Guadalajara. Guadalajara, México. Contacto: esmeralda.brito@cut.udg.mx

3 Licenciado en Administración de Empresas, maestro en Administración, candidato a doctor en Administración. Universidad Autónoma de Guadalajara. Guadalajara, México. Contacto: smagana@edu.uag.mx

4 Ingeniero civil, maestro en Ingeniería, doctor en Ciudad, Territorio y Sustentabilidad, Universidad de Guadalajara. Guadalajara, México. Contacto: mario.gonzalez@academico.udg.mx 
(negentropías); por ejemplo: heijunka, jidoka, SMED, lean manufacturing, just in time, Sistema de Producción Toyota (TPS), $5 \mathrm{~S}$ o gemba, ya que es una forma de incrementar la productividad, bajar costos y aumentar la capacidad de competitividad para atraer a más mercados internacionales.

Palabras clave: competitividad, justo a tiempo, logística, kaizen, productividad.

\section{Abstract}

Context: in Mexico, international trade is experiencing an increase joined by international companies in the manufacturing area, in the state of Jalisco. The research reviews the homeostasis of the assembly and exportation companies, with the idea of improving shipping logistics to automate and increase productivity, through the development and application of the continuous improvement tool called kaizen.

Method: this research is based on a systemic approach. In this sense, to identify the company's homeostasis, data and information were obtained on the activities carried out by the logistics area, as well as on policies and strategies. Subsequently, through the Ishikawa diagram, the cause-effect relationship was analyzed and through a process mapping the problem of the areas of purchase, production and deposit was determined. Finally, several alternative solutions were proposed to improve management. In addition, for the development of the solutions, the problems and solutions were faced by relating them to the impact on the level of service and making a comparison between before and after implementing the kaizen.

Results: The study focuses on the shipping logistics operation to automate the process and avoid bottlenecks. In this way, orders that are not sent on time become code A (urgent orders) and make the customer have a productivity rating of $60 \%$. Therefore, when implementing Kaizen, it is possible to increase considerably up to $35 \%$ per year, with $95 \%$ satisfaction and qualification samples. It is worth mentioning that this kaizen was carried out in the Fru's \& Opt project.

Conclusions: The current homeostasis of the tools mentioned in the study are basic and necessary. Also, continuous improvement tools continue to be updated and adapted; For example: Heijumka, Jidoka, Smed, Lean Manufacturing, Just in Time, Toyota Production System (TPS), 5s or Gemba, as it is a way to increase productivity, reduce costs and increase competitiveness to attract more markets International

Keywords: competitiveness, just in time, just in time, kaizen, productivity.

\section{INTRODUCCIÓN}

El comercio internacional y la tecnología son factores importantes para el desarrollo económico y social de México. Además, la globalización y el internet han logrado que el comercio internacional configure canales de comunicación, optimización en los procesos de importación, exportación, disminución de costos y tiempos de distribución de la producción de las empresas. Sin embargo, en la actualidad, "grandes empresas han optado por implementar fuentes renovables de energía" (Arizaga, González y Asprilla, 2019, p. 21), debido a la presión internacional y la concientización ambiental a nivel global. Las empresas pueden ser concebidas como sistemas constituidos por subsistemas, que consumen (inputs) y emiten materia y energía (outputs) necesarias para su funcionamiento (proceso). Además, como todo sistema, las empresas se degradan en función del tiempo (entropía) y se reestructuran en función de sus componentes intrasistémicos y las fuerzas provenientes de su entorno. No obstante, pueden implementar acciones o "fuerzas que pretenden el efecto contrario y son conocidas comúnmente como 'fuerzas neguentrópicas'" (De Quevedo, Asprilla y González, 2017, p. 140).

El entorno de las empresas suele ser competitivo y en ocasiones incierto. Esta situación complica 
aún más lograr el mantenimiento de su homeostasis en condiciones estables. Esta, según González (2018, p. 173), puede ser identificada de tres formas: a) reversible, b) cuasirreversible y c) irreversible. En la primera, el sistema tiene la propiedad de revertir afectaciones en sus elementos o relaciones, a través de la disminución de niveles de entropía. En la segunda, el sistema presenta daños parciales en algunos elementos o relaciones. En la tercera, el sistema ya no puede revertir todas las afectaciones.

En este contexto, el presente estudio revisa la homeostasis de las empresas del ramo electrónico qué implementan el kaizen para la automatización del proceso de logística de embarques. Toda vez que es conocido que, al automatizar la información de forma eficiente y eficaz, se mejoran los tiempos de respuesta y se garantiza la calidad en el servicio y atención al cliente. "Cuando se aplica al lugar del trabajo, kaizen significa un mejoramiento continuo que involucra a todos -gerentes y trabajadores por igual-" (Imai, 2001, p. 23). En este sentido, el just in time y el Sistema de Producción Toyota (SPT) son claves para alcanzar las metas, bajar costos y plazos de entrega (Blanco, Guerra, Villalpando y Castillo, 2010; Pérez, 2014).

El kaizen es la actividad que permite promocionar la mejora continua en el proceso del servicio al cliente, desde el momento en que se recibe la orden; pasa por la planeación, producción, control de calidad, almacenamiento, surtido y embarque; hasta la generación de una estructura de proceso esbelta que permita incrementar la productividad.

En las empresas internacionales dedicadas al ensamble y exportación en México, cada día implementan nuevas herramientas para el desarrollo de los procesos de automatización (Jaén y León, 2005; Suárez y Miguel, 2011; Secretaría de Economía, 2012). Muchas de estas mejoras de origen japonés están dando grandes resultados e incrementando la productividad, mayores ventas, menor costo y precio al cliente. Asimismo, "hoy en día vale la pena ver en Japón un modelo para seguir en cuanto al desarrollo sostenible" (Mesa y Villa, 2016, p. 45).).
De ahí, la implementación del kaizen permite crear procesos esbeltos que dan mejores resultados, optimizan recursos y generan mayores ganancias (Guizado y Hermoza, 2014; Ibarra y Ballesteros, 2017). Efectivamente, las exportaciones aumentan al incrementar calidad, productividad y servicio al cliente; de ahí, la importancia de realizar auditorías internas al proceso para evitar retrasos y baja productividad (Arter, 2003).

Las empresas manufactureras adquieren la mejora continua, el lean manufacturing, el kaizen, el justo a tiempo, entre otras, auxiliadas por las tecnologías de la información y comunicación (TIC), con la finalidad dar soluciones informáticas a los mercados. En este ámbito, México ha logrado un avance significativo en materia del comercio gracias a que utiliza las TIC en tratados comerciales, cultura de negocios, talento altamente calificado, geolocalización y competitividad en los costos. "En el sector logístico la cooperación entre diferentes empresas cobra especial importancia debido a la necesidad de optimizar los procesos de la cadena de suministro y reducir los tiempos de entrega" (ICIL Online, 2016).

El estado de Jalisco alberga al denominado Silicon Valley mexicano, debido a que tiene desde hace diez años a 15 grandes corporativos, principalmente de origen estadounidense. Además, cerca de 600 pymes del tipo startups, "que exportan el $70 \%$ de los materiales y contenidos que producen. Firmas como Oracle, HP, Motorola e IBM han hecho de Jalisco su segunda casa" (Corona, 2017). En 2016, el estado tenía el la posición 8 en exportaciones (USD 17,877.9 millones): es decir, el 5,5\% a nivel nacional. La principal actividad fue la fabricación de equipo de computación, comunicación, medición y de otros equipos, componentes y accesorios electrónicos (INEGI, 2017). Asimismo, Entre enero y septiembre de 2017, el estado "atrajo una inversión extranjera directa (IED) de 810.0 mdd, lo que representó el 3,7\% del total nacional" (Secretaría de Economía, 2017). Para 2018, Jalisco se ubicaba en el lugar 7 en materia de exportaciones, tal como se muestra en la figura 1. 


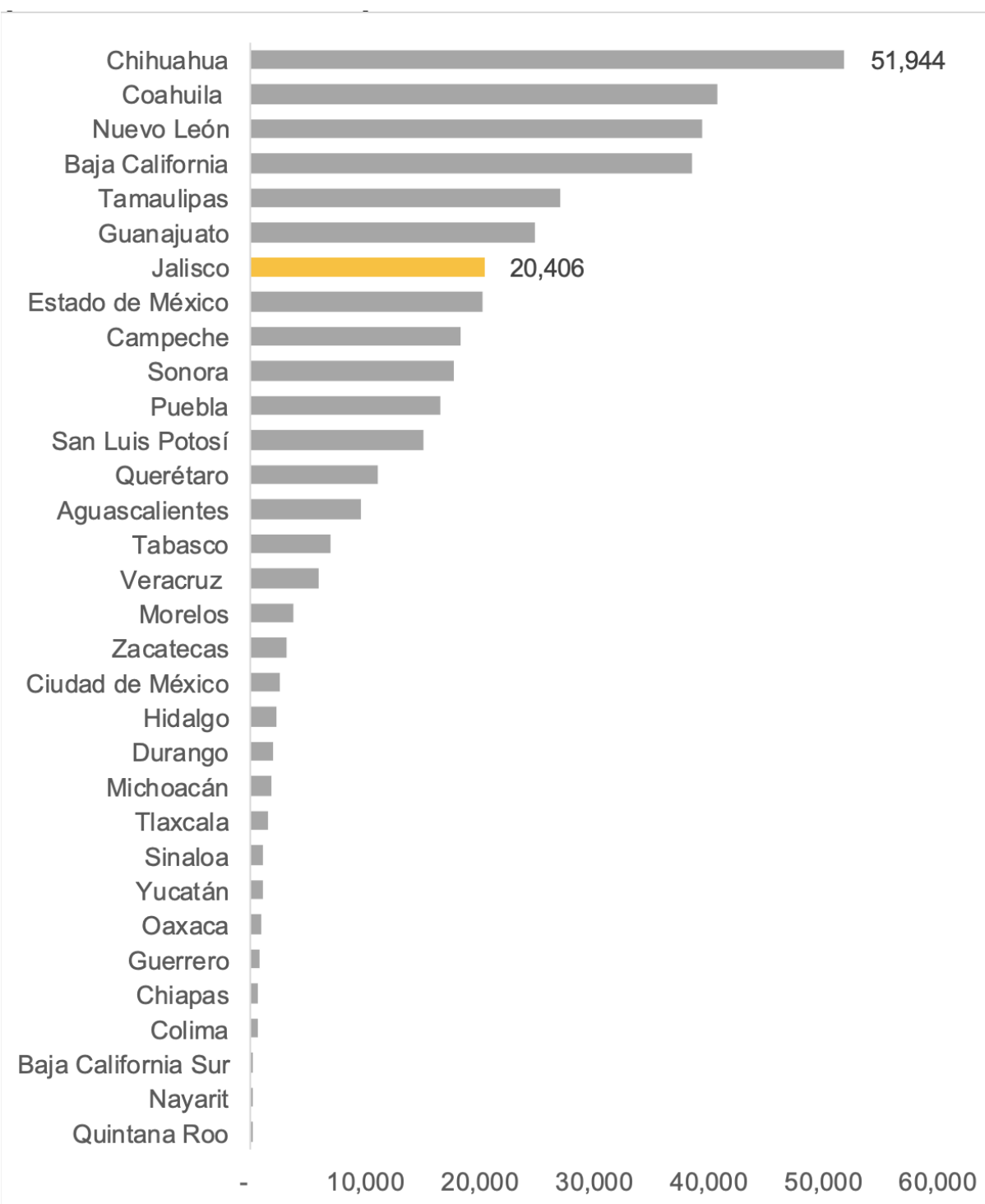

Figura 1. Exportaciones en 2018 por entidad federativa (millones de dólares)

Fuente: IIEG (2019) con datos de INEGI (2019).

\section{METODOLOGÍA}

En este estudio se analizó el problema que existía en el proyecto Fru's \& Opt (IBM) de la Empresa Flextronics. Flextronics Manufacturing de México es una de las empresas de electrónica más importante del país. "Los empleos reportados en sus registros públicos oscilan los 25 mil. Y tuvo ventas mayores a los 77 mil millones de pesos en 2017"
(García, 2018). De acuerdo con la tabla 1, en 2019, la empresa se ubicó en la posición 47 del ranking de las empresas más importantes de México.

En función de lo anterior, se elaboró un diagrama de Ishikawa (1986), para identificar las causas que originan porcentajes bajos, con la intención de implementar el kaizen para mejorar la productividad y el servicio al cliente, como se muestra en la figura 2. 
Tabla 1. Posición de Flextronics en el ranking nacional

\begin{tabular}{|c|c|c|c|}
\hline Ranking 2019* & Empresa/Estado & Sector & Ventas (millones de pesos) \\
\hline 1 & Petróleos Mexicanos & Petróleo y gas & $1^{\prime} 681.119,2$ \\
\hline 2 & América Móvil & Telecomunicaciones & $1^{\prime} 038.207,7$ \\
\hline 3 & Walmart de México & Comercio autoservicio & $612.186,0$ \\
\hline 4 & Comisión Federal de Electricidad & Electricidad & $553.358,1$ \\
\hline 5 & FEMSA & Holding & $469.744,0$ \\
\hline 6 & General Motors de México & Armadora & $418.199,0$ \\
\hline 7 & Alfa & Holding & $366.432,0$ \\
\hline 8 & FCA México & Armadora & $360.788,5$ \\
\hline 9 & Grupo Bimbo & Alimentos & $288.266,0$ \\
\hline 10 & Cemex & Cemento y materiales & $276.854,8$ \\
\hline 11 & Grupo BBVA Bancomer & Servicios financieros & $268.654,0$ \\
\hline 12 & Volkswagen de México & Armadora & $210.820,6$ \\
\hline 13 & Grupo México & Holding & $201.927,9$ \\
\hline 14 & Grupo BAL & Holding & $200,709.4$ \\
\hline 15 & Nissan Mexicana & Armadora & $196.197,3$ \\
\hline 16 & Grupo Financiero Banorte & Servicios financieros & $193.445,0$ \\
\hline 17 & Grupo Financiero Citibanamex & Servicios financieros & $185.393,0$ \\
\hline 18 & Coca-Cola FEMSA & Bebidas y cervezas & $182.342,0$ \\
\hline 19 & Kaluz & Holding & $178.569,0$ \\
\hline 20 & Honda de México & Armadora & $173.224,2$ \\
\hline 21 & FEMSA Comercio (Oxxo) & Comercio autoservicio & $167.458,0$ \\
\hline 22 & Ford de México & Armadora & $165.571,0$ \\
\hline 23 & Grupo Coppel & Comercio departamental & $161.442,0$ \\
\hline 24 & Arca Continental & Bebidas y cervezas & $158.952,5$ \\
\hline 25 & Infonavit & Servicios financieros & $157.423,0$ \\
\hline 26 & Organización Soriana & Comercio autoservicio & $153.475,0$ \\
\hline 27 & Americas Mining Corporation & Minería & $151.686,0$ \\
\hline 28 & Grupo Techint en México & Holding & $150.000,0$ \\
\hline 29 & Goldman Sachs Casa de Bolsa & Servicios financieros & $146.371,0$ \\
\hline 30 & Mexichem & Química y petroquímica & $138.492,1$ \\
\hline 31 & Grupo Financiero Santander México & Servicios financieros & $137.897,0$ \\
\hline 32 & El Puerto de Liverpool & Comercio departamental & $135.534,8$ \\
\hline 33 & Alpek & Química y petroquímica & $134.523,2$ \\
\hline 34 & Grupo Salinas & Holding & $131.684,6$ \\
\hline 35 & Sam's Club & Comercio autoservicio & $124.824,5$ \\
\hline 36 & Ternium México & Siderurgia y metalurgia & $122.443,4$ \\
\hline 37 & Sigma Alimentos & Alimentos & $121.899,8$ \\
\hline 38 & Grupo Comercial Chedraui & Comercio autoservicio & $116.030,9$ \\
\hline 39 & Grupo Elektra & Holding & $103.522,3$ \\
\hline 40 & Grupo Televisa & Telecomunicaciones & $101.282,3$ \\
\hline 41 & Grupo Carso & Holding & $96.639,8$ \\
\hline 42 & Nemak & Automotriz y autopartes & $90.327,1$ \\
\hline 43 & Samsung México & Electrónica de consumo & $90.000,0$ \\
\hline 44 & Magna International México & Automotriz y autopartes & $88.850,3$ \\
\hline 45 & Industrias Peñoles & Minería & $84.287,7$ \\
\hline 46 & Grupo Modelo & Bebidas y cervezas & $83.956,0$ \\
\hline 47 & Flextronics Manufacturing México & Electrónica & $83.924,9$ \\
\hline
\end{tabular}

Fuente: Revista Expansión (2018). 


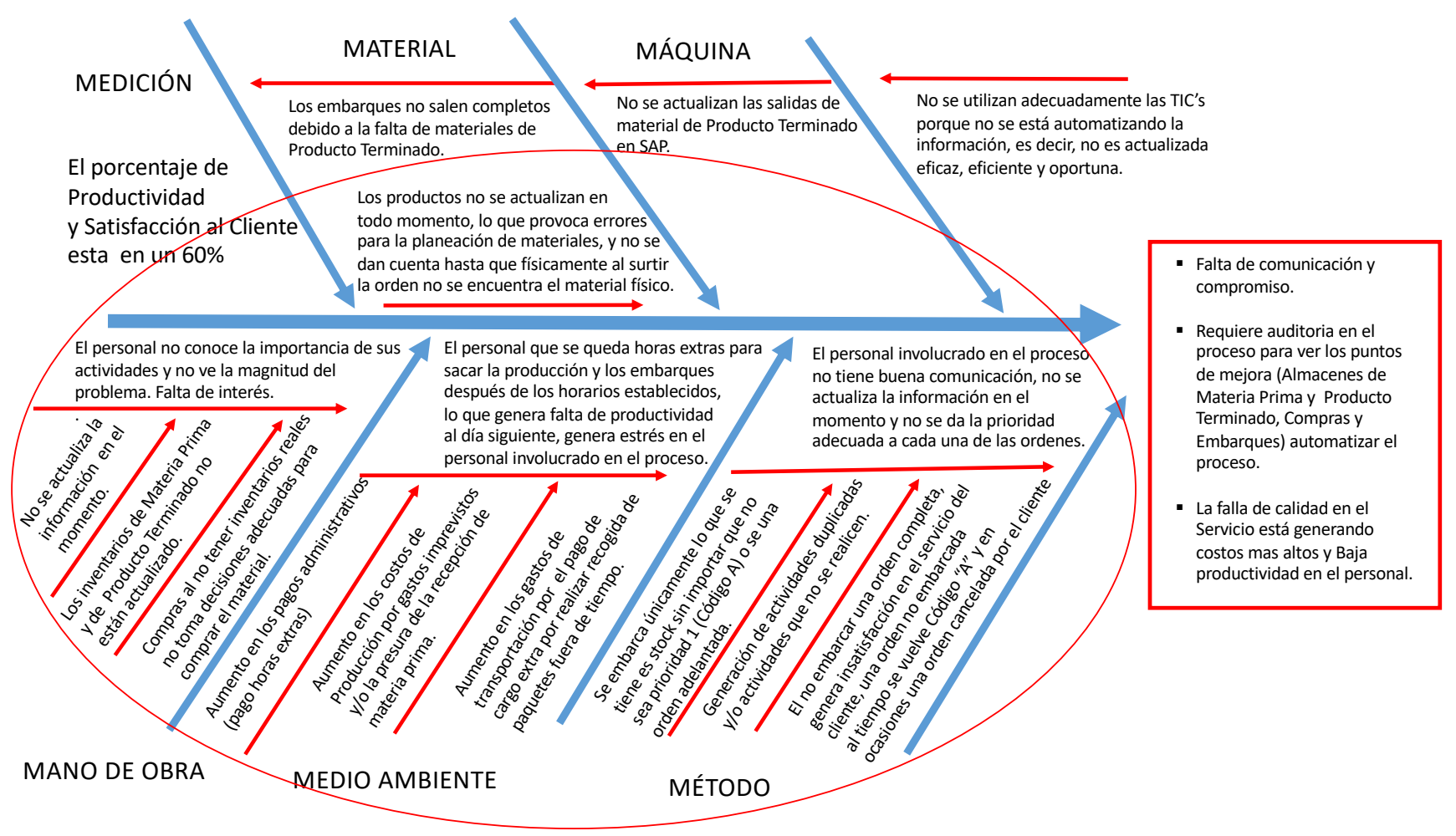

Figura 2. Aplicación del diagrama de Ishikawa (1986)

Fuente: elaboración propia.

En este estudio se toma como base la teoría de Deming (2000) sobre calidad y costos, el caso Toyota por ser la primera empresa en implementar la mejora continua mediante el desarrollo de kaizen y crear el lean manufacturing. Igualmente, son utilizadas las $5 \mathrm{~S}$ y el just in time (Piñero, Vivas y Flores de Valga, 2018; Tejeda, 2011). De esta manera, estos instrumentos posibilitan: a) El desarrollo e implementación del kaizen en la logística de embarques para automatizar los procesos e incrementar la productividad en embarques a tiempo. b) Mantener las plataformas y bases de datos con información actualizada y vinculadas en una sola, para tener información veraz y precisa en todo momento. c) Dar prioridad a las órdenes de código A. desde el momento en que se recibe la orden, se verifican los inventarios, en caso de no tener stock se debe solicitar al planeador de producción para que lo programe y se tenga a tiempo para ser embarcado. De ahí, es preciso aplicar just in time y lean manufacturing en esta fase. d) Separar las órdenes por prioridad de embarque asignándoles la etiqueta de color que identifique su prioridad e inmediatamente revisar inventarios y pasar a almacén para que se surta y se embarque. En caso de no contar con stock, es necesario avisar de inmediato a producción para que lo agregue a su plan, sin dejar de seguir las órdenes urgentes. e) Establecer una línea de embarque especial para ordenes urgentes (códigos A) con el fin de agilizar su proceso de embarque. f) Incrementar la calidad en el servicio al cliente y la productividad.

Es preciso hacer mención que Flextronics entre 2009 y2010 realizaba entre 2 y 3 kaizen por año. Actualmente en 2019 realizan de 4 a 5 kaizen por bimestre. Así, para conocer cómo operan los procesos de logística en la distribución y exportación de los componentes electrónicos a mercados internacionales, se analizan estrategias para aplicar una 
mejora continua mediante un kaizen que automatice las actividades que se llevan a cabo en cada área involucrada en el proceso. La hipótesis principal supone que la aplicación del kaizen, desde las órdenes de compra y la recepción de pedidos, es concentrada en una base de datos para generar las ordenes de compra y priorizar cada una con una etiqueta distintiva y Ilamativa para que sea identificada rápidamente por cada departamento.

En este sentido, para validar o refutar este planteamiento, se llevó a cabo una metodología basada en el análisis de los procedimientos de la logística de embarques, la distribución y los procedimientos de las áreas involucradas, con el fin de sistematizar y automatizar un procedimiento más esbelto que genere mayor flujo de la información y del sistema y los componentes.

En un segundo momento, el kaizen es implementado como una prueba piloto, con la intención de evaluar resultados y corregir errores. Durante el desarrollo del kaizen se aplicaron cuatro fases en las que se fue notando la mejora del proceso, se redujo la sobrecarga de trabajo y aumentó la calidad en el servicio al cliente. Es decir, se automatizó e incrementó la productividad en empresas internacionales que ensamblan y exportan componentes electrónicos en el estado de Jalisco.

La estrategia clave en el kaizen fue colocar una etiqueta de color a las órdenes del packing list impresas. Estas identifican la prioridad del embarque, y las personas involucradas dan la prioridad según cada color. Las ordenes de etiqueta roja representan pedidos urgentes (códigos A), los cuales se pasan directamente a la línea exclusiva de códigos A. De esta forma, se recibe la orden y se verifica el inventario; si no se dispone en stock, se solicita al planeador de producción. Igualmente, en caso de discrepancias con varios déficits, se convoca de inmediato a reunión para analizar lo sucedido y de inmediato se toman las decisiones a partir de la información que presentan los gerentes de compras, producción, almacén de materia prima, producto terminado, calidad, embarques y transportación, con el fin de no afectar el proceso de salida.

\section{RESULTADOS}

Para la aplicación del kaizen en la logística de embarques se inició con el análisis de las problemáticas experimentadas y las posibles mejoras. Asimismo, es utilizado del diagrama de Ishikawa (1986), como se indica en la tabla 2.

El modo de operación antes de aplicar el kaizen es el siguiente orden:

1. Llegan las órdenes por parte del cliente mediante una base de datos al administrador de órdenes de compra y servicio al cliente (AOCSC).

2. El AOCSC genera e imprime los packing list y entrega a embarques todas las órdenes con su packing.

3. Embarques verifica el stock y marca los productos faltantes, dejando los que tienen material faltante en una carpeta que al final entrega al AOCSC.

4. De las órdenes con material en existencia se pasan a surtido, empaque y embalaje.

5. Calidad realiza la inspección verificando los estándares de calidad.

6. Si se cumple con los estándares de calidad todo se envía a embarques y distribución, en donde se da de alta para generar las guías de embarque y posteriormente distribuir, ya sea vía terrestre o aérea.

7. Se notifica al AOCSC los números de guía con el cual se embarca cada orden.

8. AOCSC notifica a su cliente las guías con las cuales se embarcaron los componentes, así como la fecha y hora de llegada a su destino cada una.

9. El AOCSC recibe por parte de embarques las órdenes y packing que no fueron embarcadas para que se determine si la orden cambia de prioridad. Asimismo, se da aviso al planeador de producción para que contemple los componentes faltantes en su plan de producción.

10.El área de producción por su parte debe pasar a línea el plan requerido, el cual posteriormente pasa por control de calidad, para verificar que cumpla con los estándares de calidad y se entrega al almacén de producto terminado. 
Tabla 2. Resultados aplicando el kaizen

\begin{tabular}{|c|c|c|c|}
\hline Homeostasis & Replanteamiento & Kaizen & Fecha compromiso \\
\hline $\begin{array}{l}\text { Acumulación de órdenes } \\
\text { urgentes (códigos A) }\end{array}$ & $\begin{array}{l}\text { Se detectó el aumento de las órdenes } \\
\text { urgentes porque se estaban acumulando } \\
\text { aquellas que se quedaban por falta de } \\
\text { material. En ocasiones algunas fueron } \\
\text { canceladas. }\end{array}$ & $\begin{array}{l}\text { Mediante un identificador, } \\
\text { que fue la etiqueta de color, } \\
\text { se daba la prioridad a cada } \\
\text { orden. }\end{array}$ & $\begin{array}{l}\text { Desde el primer mes para } \\
\text { obtener y medir los resul- } \\
\text { tados; nueve meses para } \\
\text { alcanzar el objetivo. }\end{array}$ \\
\hline $\begin{array}{l}\text { Insatisfacción del cliente } \\
\text { al calificar con el } 60 \% \text { la } \\
\text { calidad de servicio. Por } \\
\text { tanto, la productividad se } \\
\text { ve afectada en el mismo } \\
\text { porcentaje }\end{array}$ & $\begin{array}{l}\text { Al realizar la revisión general del proce- } \\
\text { so y cada una de las áreas involucradas, } \\
\text { se detectó primero la falta de comunica- } \\
\text { ción; esto debido a que cada uno hacía } \\
\text { el Ilenado en su macro y después rea- } \\
\text { lizaba la altas o bajas en la plataforma } \\
\text { SAP. Esto trae como consecuencia que la } \\
\text { información no sea oportuna y eficiente. }\end{array}$ & $\begin{array}{l}\text { Se aplicaron revisiones alea- } \\
\text { torias para verificar que la in- } \\
\text { formación se diera en el mo- } \\
\text { mento que se realizaban las } \\
\text { altas o bajas en el sistema. }\end{array}$ & $\begin{array}{l}\text { Un mes para obtener y } \\
\text { medir los resultados. }\end{array}$ \\
\hline $\begin{array}{l}\text { Falta de comunicación en- } \\
\text { tre los departamentos in- } \\
\text { volucrados en el proceso. }\end{array}$ & $\begin{array}{l}\text { La falta de comunicación está causando } \\
\text { retrasos y desorganización en la logística } \\
\text { de embarques }\end{array}$ & $\begin{array}{l}\text { Se realizó una junta sema- } \\
\text { nal (los días lunes) para que } \\
\text { todas las áreas involucra- } \\
\text { das estuvieran enteradas del } \\
\text { material que tenía que ser } \\
\text { embarcado esa semana y la } \\
\text { siguiente; a fin de ir redu- } \\
\text { ciendo los códigos A. }\end{array}$ & $\begin{array}{l}\text { De inmediato, la comuni- } \\
\text { cación es básica para que } \\
\text { un proceso de logística de } \\
\text { embarques funcione. }\end{array}$ \\
\hline $\begin{array}{l}\text { El que no se tengan los } \\
\text { materiales en almacén } \\
\text { de producto terminado } \\
\text { a tiempo físicamente o } \\
\text { en línea, retrasa las ope- } \\
\text { raciones sucesivas para } \\
\text { completar el proceso de } \\
\text { embarques. }\end{array}$ & $\begin{array}{l}\text { Se detectó que el aumento de las órdenes } \\
\text { urgentes (códigos A) es porque el mate- } \\
\text { rial no está dado de alta en SAP, por lo } \\
\text { que se desconoce su existencia en alma- } \\
\text { cén de producto terminado. Igualmente, } \\
\text { el material que está en línea o por salir } \\
\text { de línea no capturado y actualizado en } \\
\text { el sistema causa errores como la duplica- } \\
\text { ción de órdenes. }\end{array}$ & $\begin{array}{l}\text { Mantener la información en } \\
\text { línea, automatizando la in- } \\
\text { formación con datos verídi- } \\
\text { cos, precisos. } \\
\text { Se ve la forma de mantener } \\
\text { vinculadas las bases de datos } \\
\text { individuales y de articularlos } \\
\text { a todos en estas, pero con ac- } \\
\text { cesos propios. }\end{array}$ & $\begin{array}{l}\text { Desde el primer mes para } \\
\text { obtener y medir los resul- } \\
\text { tados; nueve meses para } \\
\text { alcanzar el objetivo. }\end{array}$ \\
\hline
\end{tabular}

Fuente: elaboración propia.

11. El planeador de producción verifica que una vez terminado el producto, avise al AOCSC de que ya se encuentra listo para ser embarcado.

12.El AOCSC debe reimprimir con fecha actual la orden de embarque y el packing list, repitiendo desde el inciso a) hasta el h), como se muestra en la figura 3.

Al utilizar el proceso anterior, las órdenes de embarque se surtían, pero no en su totalidad. Algunas se demoraban en los tiempos de producción y otras se cancelaban por falta de inventarios. Así, al realizar los cambios de inventario de forma inmediata se generaban órdenes duplicadas. Por ello, al implementar el kaizen como plan de mejora, se redujeron los tiempos del proceso de logística de embarques en un $95 \%$, donde al aplicar la teoría de Deming y colocar el filtro desde un inicio, fue posible reducir tiempos de espera, incrementos de la calidad en el servicio y la productividad.

Al llegar una orden de compra se identificó la fecha en que debe ser embarcada y se asignó su prioridad. Por ejemplo, si la fecha está próxima (menos de dos días) se consideró como código A ("URGENTE"), se identificó con una etiqueta color rojo; sin embargo, si la fecha tiene un rango de entre 3 y 8 días, se consideró como "PREVIA" y se le colocó una etiqueta en color amarillo, y en 


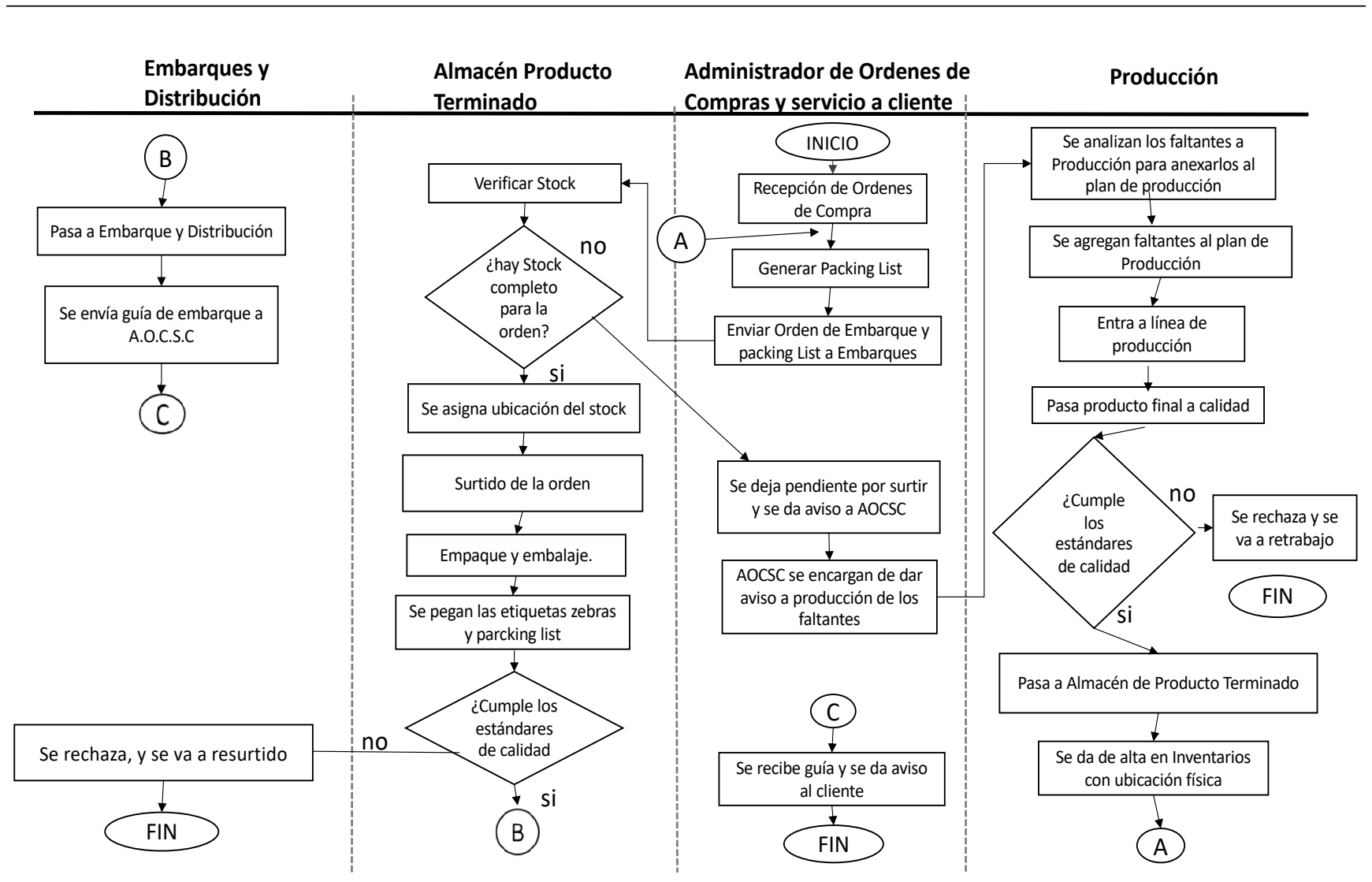

Figura 3. Proceso de embarques antes de implementar el kaizen en la logística de embarques

Fuente: elaboración propia.

ordenes con rango mayor a 8 días, se consideró como orden en "TIEMPO" y se le asignó el color verde. Esta clasificación se realizó con el fin de ser identificadas; de ahí, la elección de colores Ilamativos que permitieran distinguir la prioridad.

En el área de almacén de producto terminado se instaló una línea especial que funcionó como caja rápida; ello, con la intención de dar prioridad y velocidad correspondiente para que fuera embarcada a la brevedad. Esta actividad, durante las fases de implementación, se fue haciendo más eficiente, y se llegó a incrementar la productividad y efectividad en los embarques a tiempo; es decir, en un $35 \%$, de un $60 \%$ se elevó al $95 \%$ porque poco a poco las órdenes de códigos A fueron bajando, debido a que el cliente ya no tenía órdenes urgentes, al estar recibiendo el producto en tiempo. Inclusive, antes del tiempo estimado.
El proceso después de la implementación del kaizen mostrado en la figura 4 se estructuró en las siguientes fases:

a. Recepción de órdenes de compra mediante la base de datos que envía el cliente y se relaciona con la de la empresa, con el propósito de verificar los tiempos y asignar la prioridad del embarque.

b. Se coloca la etiqueta roja de identificación de prioridad; esta es una orden que desde su llegada debe ser monitoreada en todo momento hasta su embarque. La etiqueta amarilla se monitorea por área, y la verde, al momento de ser embarcada.

c. Se hace la verificación del stock para surtir la orden; si el inventario completa la orden, se genera el packing list y las etiquetas zebra, las cuales se envían de inmediato al área de almacén de producto terminado para que sea atendida la orden 
en su totalidad. De no estar completo el material, se da aviso al planeador de producción para que anexe al plan los componentes o piezas faltantes.

d. El almacén de producto terminado recibe el packing list, la orden y etiquetas zebra y asigna ubicaciones para que se surta. Igualmente, se identifica la prioridad y se separan para que las órdenes de etiqueta roja se pasen a la línea especial. Una vez se termina, se da aviso al AOCSC para que de inmediato se contacte con embarques y distribución, y así obtener el número de guía para proporcionárselo al cliente. Con las prioridades amarilla y verde se pasan normales a surtido.

e. Se empacan; algunas pasan por embalaje y se colocan las etiquetas zebra, el packing list y son auditadas por calidad para verificar que cumplan con los estándares requeridos. Si cumple con todo, se pasan al área de embarque y distribución, donde se les subdivide según el peso, tamaño, lugar de destino y número de guía. Para el embarque y exportación, entra la gestión aduanal con base en los requerimientos y especificaciones del cliente. En caso contrario (incumplimiento de los estándares de calidad requeridos), se rechaza para que se revise de nuevo y se realicen las adecuaciones necesarias-

f. Una vez que se embarca, los números de guía se envían al AOCSC para que este proporcione la confirmación al cliente. Así, se aumenta la productividad en un $95 \%$ y se reducen los tiempos muertos o de espera en un $85 \%$.

En el presente estudio, se aplicó un kaizen en la logística de embarques para automatizar e incrementar la productividad en el proyecto Fru's \&

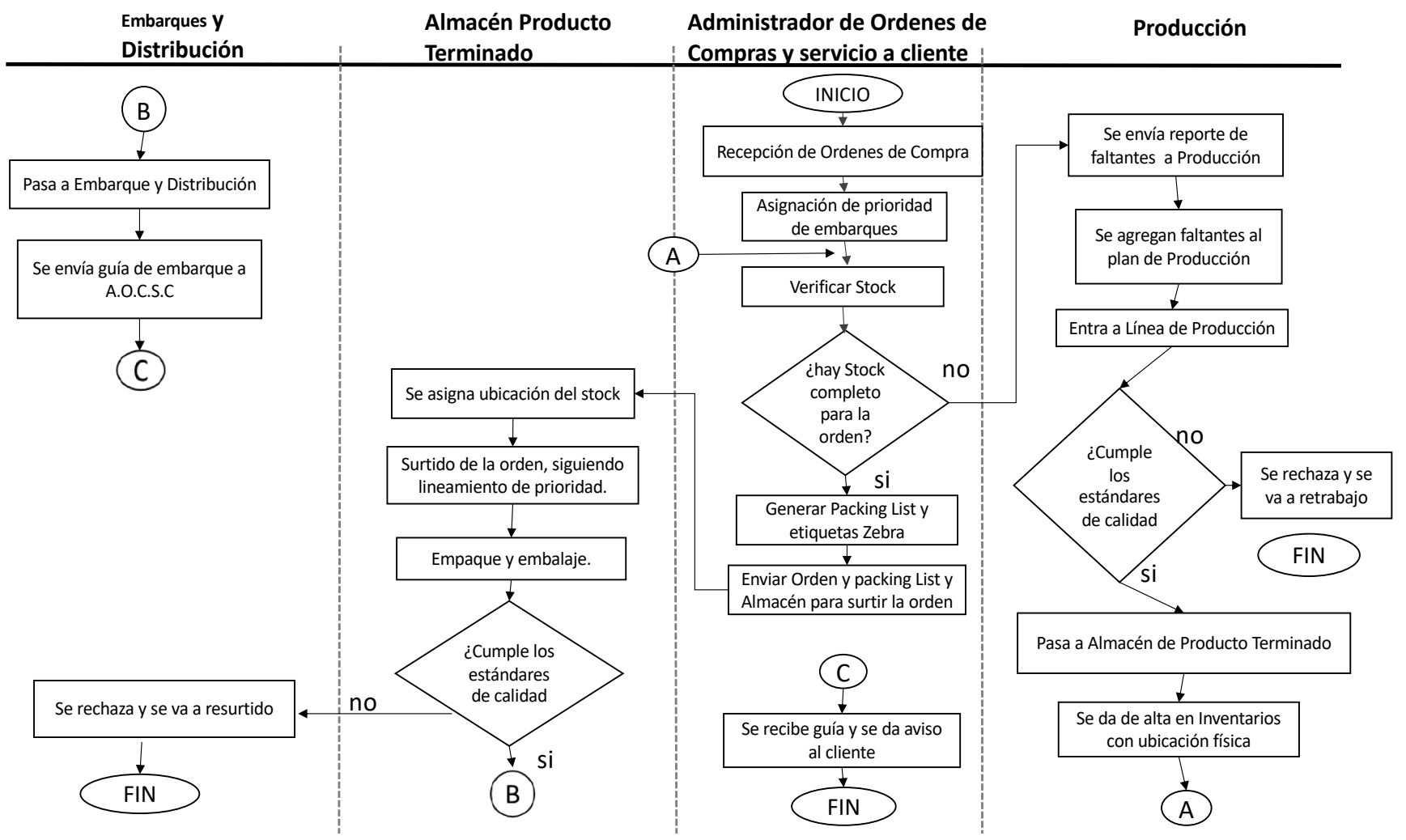

Figura 4. Proceso de embarques después de implementar el kaizen en la logística de embarques

Fuente: Elaboración propia 
Opt. En ese sentido, automatizar las operaciones en el área de logística de embarques implica tener la información de manera eficiente y eficaz. Sobre todo, porque el comercio internacional requiere tiempos precisos para el éxito de los proyectos de exportación. Se cumplió, entonces, con el supuesto inicial -cuyo argumento sostiene que al implementar el kaizen de automatización en el proceso de logística de embarques, se reducen los tiempos de operación-, porque se dio mayor agilidad tanto al surtido (distribución), como al embarque de cada uno de los packing list. El proceso se estructuró de forma que se redujeron tiempos muertos; además, se agilizó cada orden; concretamente, a los códigos A. Estos se redujeron al colocar la línea especial para embarques urgentes.

El proceso se modificó al hacer pequeños ajustes. El primero consistió en una reducción mayor del tiempo de espera, debido a que cuando llegaban las órdenes a la plataforma, el AOCSC las ordenaba, separaba e identificaba con la etiqueta de prioridad. El segundo ajuste fue verificar el stock, esto ayudó al tiempo de respuesta, porque al detectar la falta de un material se daba aviso de inmediato al planeador de producción para que lo agregara al plan de producción y así embarcarlo a la brevedad. El tercer ajuste fue colocar una línea en el área de embarques (almacén de producto terminado) para que se surtieran de forma independiente y con mayor supervisión y velocidad. La implementación de la línea rápida para códigos A permitió la reducción de las órdenes canceladas. Esto eliminó el sistema PUSH (apagafuegos).

Al modificar el diagrama de procesos y aplicar el lean manufacturing, se redujeron los tiempos en aproximadamente de 10 minutos de cada packing list. En el caso de Fru's \& Opt se obtiene un incremento del $35 \%$, llevando de un $60 \%$ a un $95 \%$ la productividad y calidad de servicio al cliente. Además, se logró mantener una comunicación eficaz entre los integrantes del desarrollo del kaizen; así, esta se vuelve un factor clave para lograr los objetivos, tanto con el personal interno de planta, como el personal externo y el cliente.
El just in time también fue clave en la gestión de las operaciones con el cual se logró alcanzar las metas, incrementando la calidad en todo el proceso. Se redujeron los costos de producción, así como los plazos de entrega, mediante el compromiso de las personas. El sistema jidoka benefició al stock de almacén de producto terminado, ya que al eliminar retrabajos la producción ejecuta y completa el plan de producción sin contratiempos. Esto generó flujo de materiales y los códigos A únicamente se convirtieron en órdenes especiales del cliente las cuales solicitaba y embarcaban el mismo día.

Al obtener estos resultados cuando el cliente volvió a evaluar el servicio y la productividad dio la calificación máxima del $95 \%$ dejando el 5\% como margen para continuar trabajando en la mejora continua en áreas involucradas al proceso de embarques, como compras y planeación. Un reto para alcanzar el $100 \%$ en el siguiente trimestre.

Los almacenes tenían conteos cíclicos y en cuanto se detectaba un error se corregía de inmediato, y compras verificaba con producción y embarques, qué era lo que se tenía por embarcar. De ahí, se analizaban los inventarios para verificar el stock de almacén de producto terminado con el fin de identificar qué se debía comprar y en qué fechas se planeaba la producción, según las fechas de entrega y recepción de los proveedores.

\section{CONCLUSIONES}

La aplicación del kaizen permitió mayor productividad y calidad en los procesos. Esto representa grandes oportunidades de desarrollo para las empresas, en general, y a la industria electrónica, en particular. En el periodo 2009-2010 se implementaban de 3 a 4 kaizen por año en la empresa caso de estudio. Actualmente (2019), se implementan de 3 a 4 kaizen por bimestre. Esto demuestra que la mejora continua favorece la productividad, la reducción de costos, la optimización de recursos, el tiempo y la mayor rentabilidad del proyecto. El kaizen fue la actividad que configuró cambios 
significativos, desde el momento en que se recibe la orden y pasa por el proceso de planeación, producción, control de calidad, almacenamiento, surtido y embarque; hasta la generación de una estructura de proceso esbelta. De ahí, empresas internacionales dedicadas al ensamble y exportación cada día implementan nuevas herramientas para el desarrollo de procesos de automatización, entre otras técnicas japonesas que están dando grandes resultados e incrementando la productividad y competitividad.

En este contexto, es preciso destacar la importancia de las auditorías internas en el proceso. Estas arrojan información valiosa de la homeostasis de la empresa, como dar una alta o baja a tiempo y, así, evitar retrasos y baja productividad. Por ello, las empresas que se certifican pueden ser mayormente competitivas en los mercados internacionales. El comercio internacional no solo consiste en exportar un producto, sino llevar a cabo procesos robustos que generen automatización y velocidad, para lo cual, la tecnología es un gran aliado porque facilita la apertura de nuevos mercados.

\section{REFERENCIAS}

Arizaga B., A., González P., M.G. y Asprilla L., Y. (2019). Sistemas piezoeléctricos en el tren urbano de Guadalajara, México: entropía y negentropía. Tecnura, 23(61), 13-22. https://doi. org/10.14483/22487638.14870

Arter D., R. (2003). Auditorias de calidad para mejorar la productividad. Milwaukee, Wisconsin: ASQ Quality Press.

Blanco, M., Guerra S., Villalpando, P. y Castillo J. (2010). La evolución de la industria del sector automotriz en Japón. InnOvaciOnes de NegOciOs, 7(1), p. 1-24. Recuperado de http://eprints.uanl. mx/12527/1/A1.pdf

Corona, S. (2017). El Silicon Valley mexicano está en Jalisco. ElPaís.com. Recuperado de https:// elpais.com/tecnologia/2017/03/12/actualidad/1489275848_767120.html
Deming W., E. (2000). Out of the crisis. Cambridge, MA: The MIT Press.

De Quevedo, F., Asprilla, Y. y González, M. (2017). Entropías de la movilidad urbana en el espacio metropolitano de Guadalajara: transporte privado y calidad del aire. Tecnura, 21(53), 138-149. https:// doi.org/10.14483/22487638.10725

García, O. (2018). Las 21 empresas en Jalisco, ubicadas entre las 500 más importantes de México. Recuperado de http://udgtv.com/noticias/ empresas-de-jalisco-mas-importantes-mexico/

González, M., G. (2018). Entropy and negentropy of private electric vehicles in urban systems: homeostasis of mobility in Mexico. DYNA, 85(206), p. 171-177. https://doi.org/10.15446/dyna.v85n206.72509

Guizado A., F. y Hermoza, A. (2014). Sistema de control de inventario aplicando los métodos $A B C$, Just In Time y Poka Yoke en una empresa de confecciones. En VI Congreso Internacional de Computación y Telecomunicaciones. Perú. Recuperado de http://repositorio.uigv.edu.pe/bitstream/handle/20.500.11818/728/paper_sistemadecontrol. pdf? sequence $=1$ \&isAllowed $=y$

Ibarra V., M. y Ballesteros L., L. (2017). Manufactura esbelta. Conciencia Tecnológica, 53(1), 1-8. Recuperado de http://www.redalyc.org/articulo. oa?id=94453640004

ICIL Online (2016). ¿Qué es un clúster logístico? Ventajas y desventajas. Recuperado de http://www.icilonline.com/actualidad/ cluster-logistico-ventajas-desventajas/1645/

Ishikawa, K. (1986). ¿Qué es el control total de calidad? La modalidad japonesa. Bogotá: Grupo Editorial Norma.

Instituto de Información Estadística y Geográfica de Jalisco (IIEG) (2019). Las exportaciones de Jalisco en el cuarto trimestre de 2018 en su máximo histórico. Ficha informativa. Recuperado de https://iieg.gob. mx/contenido/Economia/Ficha\%20informativaExportaciones\%2020190329.pdf

Imai, M. (2001). Kaizen. La clave de la ventaja competitiva japonesa. México: Compañía Editorial Continental, S.A. de C.V. 
Instituto Nacional de Estadística, Geografía e Informática (INEGI) (2017). Banco de Información Económica. Estadísticas de Exportaciones por Entidad Federativa. Recuperado de: http://www.beta.inegi. org.mx/datos/

Instituto Nacional de Estadística, Geografía e Informática (INEGI) (2019). Balanza comercial de México. Recuperado de https://www.inegi.org.mx/temas/ balanza/default.html\#Informacion_general

Jaén, B. y León, M. (2005). La industria electrónica de exportación en Jalisco, México. Comercio Exterior, 3(55), 270-281. Recuperado de http://revistas.bancomext.gob.mx/rce/magazines/76/6/RCE.pdf

Mesa S., C. y Villa M., A. (2016). La política ambiental japonesa y la movilización de industrias por la protección ambiental. Revista Mundo Asia Pacífico, 8(5), 43-50. https://doi.org/10.17230/map.v5.i8.04

Pérez, J. (2014). Just in time aplicado en la industria de la construcción. [Tesis de especialización en ingeniería]. Universidad Nacional Autónoma de México, México. Recuperado de http://www.ptolomeo.unam.mx:8080/jspui/bitstream/132.248.52.100/6922/1/TESINA\%20 JUST\%20IN\%20TIME\%20pdf.pdf

Piñero, E., A, Vivas F., E. y Flores de Valga, L.K. (2018). Programa $5 S$ para el mejoramiento continuo de la calidad y la productividad en los puestos de trabajo. Ingeniería Industrial. Actualidad y Nuevas
Tendencias, 6(20), 99-110. Recuperado de http:// servicio.bc.uc.edu.ve/ingenieria/revista/Inge-Industrial/volvi-n20/art06.pdf

Revista Expansión (2019). Las 500 empresas más importantes de México. Recuperado de: https:// expansion.mx/empresas/2019/06/28/estas-son-las500-empresas-mas-importantes-de-mexico-2019

Secretaría de Economía (2012). Industria automotriz. Monografía Dirección General de Industrias Pesadas y de Alta Tecnología. Recuperado de: https:// www.economia.gob.mx/files/comunidad_negocios/industria_comercio/Monografia_Industria_Automotriz_MARZO_2012.pdf

Secretaría de Economía (2017). Competitividad y normatividad. Inversión extranjera directa. Recuperado de https://www.gob.mx/se/acciones-y-programas/ competitividad-y-normatividad-inversion-extranjera-directa? state $=$ published

Suárez M., F. y Miguel J., A. (2011). Implementación del kaizen en México: un estudio exploratorio de una aproximación gerencial japonesa en el contexto latinoamericano. Innovar. Revista de Ciencias Administrativas y Sociales, 41(21), 19-37. Recuperado de https://www.redalyc.org/pdf/818/81822806003.pdf

Tejeda, A.S. (2011). Mejoras de lean manufacturing en los sistemas productivos. Ciencia y Sociedad, 36(2), 276-310. https://doi.org/10.22206/cys.2011.v36i2. pp276-310 